Conclusions Genetic deletion or pharmacological inhibition of NOX2 sensitized AML cells to daunorubicin induced killing in hypoxic environments. NOX2 may thus be a target for overcoming chemoresistance in AML cells in the hypoxic bone marrow environment.

Support This work was supported by Assar Gabrielsson's Foundation( FB19-64) and other grants used by the research group.

Disclosure Information S. Paul: None. H. Grauers Wiktorin: E. Ownership Interest (stock, stock options, patent or other intellectual property); Modest; Patent. R. Kiffin: None. K. Hellstrand: E. Ownership Interest (stock, stock options, patent or other intellectual property); Modest; Patent. A. Martner: E. Ownership Interest (stock, stock options, patent or other intellectual property); Modest; Patent.

\section{P03.28 STRUCTURAL CHARACTERISTICS IN TUMOR AND LYMPH NODES AS PREDICTORS OF 3-YEAR METASTASIS-FREE SURVIVAL IN SURGICALLY TREATED NSCLC}

${ }^{1} \mathrm{~L}$ Sellmer* ${ }^{2}{ }^{2} \mathrm{~J}$ Kovacs, ${ }^{3} \mathrm{~J}$ Neumann, ${ }^{1} \mathrm{R}$ Kiefl, ${ }^{1} \mathrm{D}$ Kauffmann-Guerrero, ${ }^{2} \mathrm{C}$ Schneider, ${ }^{1}$ A Tufman. ${ }^{1}$ Department of Thoracic Oncology, Hospital of the Ludwig-Maximilians University, Munich, Germany; ${ }^{2}$ Department of Thoracic Surgery, Hospital of the LudwigMaximilians University, Munich, Germany; ${ }^{3}$ Department of Pathology, Ludwig-Maximilians University, Munich, Germany

\subsection{6/jitc-2020-ITOC7.66}

Background Surgery is the treatment of choice for early and for some locally advanced non-small cell lung cancer (NSCLC). Ipsilateral hilar and mediastinal lymph nodes are generally removed at the time of tumor resection and assessed for tumor infiltration. However, in particular in the context of immunotherapy, there is now increased awareness about the physiological role of lymph nodes in cancer. It may be possible to assess immune response by examining the cellular composition of locoregional lymph nodes. We aimed to assess structural characteristics in tumor tissue and affected and unaffected lymph nodes in patients with and without 3-year metastasis-free survival.

Materials and methods Internal hospital databases were screened for NSCLC patients fulfilling inclusion criteria. Data on patients age, sex, surgery type, (neo)adjuvant therapy, tumor characteristics and time and location of relapse was extracted. FFPE tissue blocks of primary tumor, affected and unaffected lymph nodes were collected. Hematoxylin and eosin stainings were obtained and tissues were analyzed (e.g. for B-cell proliferation and macrophage infiltration) in collaboration with an experienced pathologist.

Results A total of 754 NSCLC patients were screened for inclusion criteria. Of these, 71 patients remained in remission for at least 3 years after surgery, and 80 patients had local or systemic relapse within 3 years after surgery. Structural characteristics in tumor and lymph node immune populations differed between patients with and without 3-year metastasis-free survival.

Conclusion Structural characteristics differ between patients with and without relapse. Our findings show that structural markers in tumor and lymph nodes should be taken into account when assessing patient prognosis and relapse risk.

Disclosure Information L. Sellmer: None. J. Kovacs: None. J. Neumann: None. R. Kiefl: None. D. Kauffmann-Guerrero: None. C. Schneider: None. A. Tufman: None.

\section{P03.29 CHARACTERIZATION OF TREATMENT-INDUCED ADAPTIVE IMMUNE RESPONSES IN PANCREATIC DUCTAL ADENOCARCINOMA}

${ }^{1,2}$ J Heetmeyer ${ }^{*},{ }^{1,2} \mathrm{C}$ Falcomatà, ${ }^{1,2} \mathrm{~S}$ Bärthel, ${ }^{2,3} \mathrm{C}$ Schneeweis, ${ }^{1,2} \mathrm{~A}$ Coluccio,

${ }^{1,2} \mathrm{C}$ Veltkamp, ${ }^{2,3} \mathrm{G}$ Schneider, ${ }^{1,2} \mathrm{D}$ Saur. ${ }^{1}$ Institute of Translational Cancer Research and Experimental Cancer Therapy, TranslaTUM, Technical University Munich, München, Germany; ${ }^{2}$ German Cancer Consortium (DKTK), German Cancer Research Center (DKFZ), Heidelberg, Germany; ${ }^{3}$ Department of Internal Medicine II, Klinikum rechts der Isar, Technical University Munich, München, Germany

\subsection{6/jitc-2020-ITOC7.67}

Background Pancreatic ductal adenocarcinoma (PDAC) is a highly aggressive malignancy marked by poor prognosis and profound drug resistance characterized in more than $90 \%$ of cases by KRAS mutations. To recapitulate central aspects of PDAC, we employed genetically engineered mouse models presenting Kras ${ }^{\mathrm{G} 12 \mathrm{D}}$ pancreas specific expression. Through a high-throughput combination drug screen with trametinib as backbone we identified a high synergism with the multikinase inhibitor nintedanib, preferentially in mesenchymal PDAC, a subtype of this disease characterized by poor prognosis and therapeutic resistance. This combinatorial treatment, that led to the induction of apoptosis in vitro and disease regression in vivo, was accompanied by a strong tumor infiltration of CD8 positive $\mathrm{T}$ cells.

Materials and Methods To characterize the treatment-induced adaptive immune cell infiltration in vivo, we performed orthotopic transplantations of KRAS-driven murine PDAC cell lines presenting mesenchymal and epithelial morphology. The derived control and nintedanib + trametinib treated PDAC tumors were analyzed by multi-color immunofluorescence stainings. We compared the findings to high parameter flow cytometry results.

Results Confocal microscopy of the immunofluorescence stainings revealed an overall increase of tumor-infiltrating lymphocytes (TIL) in the tumors upon combinatorial treatment with substantial differences in quantity and spatial distribution. Tumors derived from a PDAC cell line of epithelial morphology were characterized by few TIL mainly located at the invasive margins of the tumors, while tumors derived from a mesenchymal PDAC cell line showed a strong increase of TIL even in the center of the tumor mass. Furthermore, an increased ratio of CD8 positive cytotoxic T cells to CD4 positive helper $\mathrm{T}$ cells as well as a decrease of Foxp3 and CD4 positive regulatory $\mathrm{T}$ cells could be observed for tumors derived from the mesenchymal PDAC cell line under combinatorial treatment. To investigate if the observed recruitment of $\mathrm{T}$ cells was indispensable for treatment efficacy of the combinatorial therapy, we orthotopically transplanted the mesenchymal PDAC cell line in immunodeficient CD3-Knockout (CD3ko) mice and applied an analogous combinatorial treatment scheme. In the CD3ko mice, the combinatorial treatment did not lead to an increased survival or tumor regression as observed in immunocompetent mice. However, flow cytometry and immunofluorescence stainings revealed an increase of $\mathrm{B}$ cells upon nintedanib + trametinib treatment.

Conclusions Our findings indicate a reduced efficacy of the combinatorial treatment in $\mathrm{T}$ cell deficient mice, underlining the importance of $\mathrm{T}$ cells in treatment-induced anti-tumor responses and enlarging the understanding of the role of TIL in PDAC.

Disclosure Information J. Heetmeyer: None. C. Falcomatà: None. S. Bärthel: None. C. Schneeweis: None. A. Coluccio: None. C. Veltkamp: None. G. Schneider: None. D. Saur: None. 